[zu] folgen« (a.a.O.: 39f), der »bereits eine Krankheit, ein Niedergang, ein Symptom der Erschöpfung « ist (ebd.). Diese inspirierende Müdigkeit »als Kunst des Gelassen-Seins, das heißt, dem Sein näher als dem Gestell und dem Gerede des Man, wie Heidegger sagen würde« (Böhme 2018: 28), eröffnet neue Denkräume, erschließt Möglichkeiten, die sich im Leistungs- und Wachstumsmodus erschöpft haben. Mit Gilles Deleuze gesprochen, kündigt die Erfahrung der Ohnmacht als inspirierende Müdigkeit eine Politik des Schweigens an:

»Die Schwierigkeit ist heute nicht mehr, dass wir unsere Meinung nicht frei äußern können, sondern Freiräume der Einsamkeit und des Schweigens zu schaffen, in denen wir etwas zu sagen finden. Repressive Kräfte hindern uns nicht mehr an der Meinungsäußerung. Im Cegenteil, sie zwingen uns sogar dazu. Welche Befreiung ist es, einmal nichts sagen zu müssen und schweigen zu können, denn nur dann haben wir die Möglichkeit, etwas zunehmend Seltenes zu schaffen: Etwas, das es tatsächlich wert ist, gesagt zu werden.« (Deleuze 1995: 129 zit.n. Hardt \& Negri 2013: 21)

Erschöpfung macht schweigend. Nicht einmal den Schrei des Ärgers und der Wut lässt der kraftlose Zustand der Burnout-Betroffenen zu. Und doch, das ohnmächtige Schweigen und die provozierende Stille machen das Gegenüber, das immanente Machtregime, hilflos. Was ihm bleibt, ist die Zuflucht »bei reinem Zwang oder schlichter Gewalt« (Foucault [1982] 2005: 287) zu suchen. Ohnmacht als Ausdruck der Freiheit zu verstehen, die sich der Machtausübung widersetzen will (vgl. ebd.), heißt dann, die Macht über sich selbst als Zielscheibe und Produktionsfaktor des immanenten (neoliberalen) Regierens nicht abgeben zu wollen.

\title{
3 Regieren durch Vorbeugen
}

Als drittes haben sich die kritischen Auseinandersetzungen mit der BurnoutPrävention darum bemüht, die Mechanismen des Regierens durch Vorbeugen zu entschlüsseln und dessen charakteristische Wesenszüge zu hinterfragen. Diese lassen sich auf der Mikro- und Makroebene beobachten sowie anhand von drei Aspekten - die Zeit, der Körper und das Nicht-Existente - unterscheiden.

\subsection{Regieren auf der Mikroebene}

Wie die Analysen nahegelegt haben, wirkt das immanente Regierungsregime der Prävention vor allem auf der individuellen Mikroebene. Die Subjekte der Prävention werden produziert, angesprochen, angeleitet und präventiert bzw. zur SelbstPrävention geführt. Hier wird die Verschränkung mit anderen Dispositiven bzw. ihren Teilen besonders anschaulich. Beispielhaft zeigt das Niklas Rose in seiner 
Studie zur Politik des Lebens (vgl. Rose 2009). Die neuen wissenschaftlichen Methoden und die damit einhergehende Untersuchung des menschlichen Körpers, menschlicher Psyche, menschlicher Gene, der individuellen Lebensweisen sowie der sozialpolitischen und kulturellen Rahmenbedingungen der Individuen haben ein Wissenskonglomerat hervorgebracht, welches das ganze Leben des Individuums vereinnahmen, überschauen, regulieren, kontrollieren, überwachen, modifizieren und führen kann. An dieses Wissenskonglomerat knüpfen die präventiven Praktiken auf eine Art und Weise an, die einer Abnormalität, einer Gefährdung bzw. einer Kontingenz vorbeugen kann. So interveniert bspw. Biokriminologie durch die Logik des Verdachts, des Vorhersehens und der Prävention, indem sie Anti-Bürger antizipiert, die vermehrt die Selbstkontrolle verlieren und dadurch eine gesellschaftliche Bedrohung darstellen, die auf doppelte Weise - durch das Verstehen der Bedingungen, die zu solchem Verhalten führen, und der damit einhergehenden Identifikation von Risiko-Bürgern einerseits und durch den Schutz der Bevölkerung vor den Einflüssen auf die psychische und physische Gesundheit, die von diesen Bürgern ausgehen können, andererseits - präventiert werden kann. Somit entsteht eine Wissenslücke bzw. eine politische Rationalität, die aus verschiedenen Quellen gefüllt und für Regierungszwecke brauchbar gemacht werden kann:

»Within this problem space, a new research program on the biology, neurology, neurochemistry, and genetics of crime has taken shape, seeking to locate biological processes, genetic markers, and risk factors for aggressive and antisocial conduct, and to develop techniques for identifying risky individuals. Through adoption studies, hormonal research, neurophysiological studies, and studies of intellectual ability, attention deficit disorder, and minimal brain dysfunction, researchers are searching for links between specific biological abnormalities and the propensity to commit violent crime, with a view to early identification, preventive intervention, and effective treatment. It is in these terms that a new molecular biopolitics of control is taking shape.«(a.a.O.: 241f)

Diese neue Biopolitik der Kontrolle braucht die präventive politische Rationalität, damit ihre Anwendungsmöglichkeiten ständig erweitert, optimiert und legitimiert werden können und sie sich in einer immanenten Regierungsweise auflösen kann. Auf der Mikro-Ebene besteht der Wesenszug des Regierens durch Vorbeugen darin, die bis in die Gene und tiefsten Eingeständnisse des Individuums eingedrungene Macht durch präventive Eingriffe $\mathrm{zu}$ instrumentalisieren.

\subsection{Regieren auf der Makroebene}

Die Makroebene des Wirkungsmechanismus des Präventionsregimes bezieht sich auf die Kehrseite des Neoliberalismus, die nicht nur die individualisierende und 
aktivierende Dimension enthält, sondern auch selektive und rassistische Auswirkungen auf die Bevölkerung(-en) ausübt (vgl. Schultz 2011: 242). Diese Dimension des Regierens beleuchtet bspw. Susanne Schultz, die in dem Neo-Malthusianismus eine Idee erblickt, welche die Population als eine Biomasse ansieht, die mit den immer knapper werdenden Erdressourcen in einem Spannungsverhältnis steht und damit eine justierbare Regierungs-Variable darstellt (vgl. a.a.O.: 251).

In Verbindung mit Gender-Policies, die während der Post-Cairo Periode entstanden sind und die sich auf die Reduktion der globalen Weltbevölkerung konzentriert haben (vgl. a.a.O.: 244), werden dann solche Politiken betrieben, die sich die reproduktive Gesundheit gewisser Populationsgruppen als Anwendungsgegenstand wählen, an dem die neo-malthusianische Sorge um das Überleben der globalen Menschheit vollzogen werden kann. Auch in diesem Fall wird ein MachtWissens-Nexus formiert, indem sich das epidemiologische Wissen um Müttersterblichkeit, um antinatalistische Strategien oder um die reproduktive Gesundheit unterschiedlicher Frauengruppen mit dem demografischen Ziel eines nachhaltigen Bevölkerungswachstums verbinden. Eine von solchen Politiken war die Deutsche Initiative Safe Motherhood, die sich auf die Müttersterblichkeit in den Ländern der Dritten Welt konzentriert hat (vgl. a.a.O.: 258). Im Fokus dieser Initiative steht dabei die Prävention von (Risiko-)Geburten, mit der die Zielgruppen identifiziert, Maßnahmen passgenau entwickelt und Populationen reg(-ul-)iert werden könnten: "Prevention of >reproductive risks is a paradigm on the basis of which the population establishment has identified a variety of risk regions, risk groups and risk factors in order to determine which women at what age in which situations face an increased risk of maternal mortality.« (a.a.O.: 259 [Herv. i. O.]) In die Verbindung solcher demografischen Strategien mit den neo-malthusianischen epidemiologischen Parametern tritt die Prävention als ein Heilmittel für die uneingeschränkte Populationsverbreitung einerseits und die sichernde Müttersterblichkeitsregulierung, vor allem bei den jungen Erwachsenen, andererseits. Der Wesenszug des Regierens durch Vorbeugen auf der Makro-Ebene zeigt sich in der subtilen Kombination der sexuellen Aufklärung und Emanzipation unentwickelter Regionen mit den gleichzeitigen Strategien sexueller Abstinenz, die sich an kulturellen und religiösen Haltungen orientieren (vgl. a.a.O.: 260). Hier tritt die Prävention als Aufklärungsund Regierungsprojekt zugleich auf und ermöglicht eine globale biopolitische Hegemonie.

\subsection{Beherrschung der Zeit}

Die Prävention, wie mehrmals in der Untersuchung erwähnt, zielt auf ein mehr oder weniger mögliches Auftreten eines gefährdenden Ereignisses. Dieses Ereignis wird damit stets in der Zukunft verortet, womit die Prävention den zeitlichen Aspekt des zu behandelnden Problems thematisieren muss: »Der mögliche Scha- 
densfall in der Zukunft fesselt die gegenwärtige Aufmerksamkeit, er lässt Befürchtungen entstehen und drängt zum Handeln." (Leanza 2017: 13) Die Zeit erscheint dann zum einen als ein Parameter, der darüber entscheidet, wie erfolgreich oder misslungen eine präventive Maßnahme sein wird, d.h. ob das Problem frühzeitig und ausreichend genug präventiert wurde, und zum anderen als eine bewegliche Konstante, als eine Kontingenz, die gebändigt werden muss, die aber trotz aller Bemühung nie vollständig überschaut und umfasst werden kann. Damit ruft die Prävention eine Dynamik ins Leben, die einerseits stets wachhält, andererseits aber keine Garantien bietet.

Mehr noch, die Prävention privatisiert und individualisiert die Zeit. Den Subjekten wird vermittelt, dass sie ihre Zeit sinnvoll, d.h. gesundheitsfördernd verbringen sollen - entweder durch sportliche Aktivitäten, durch einen ausreichenden Schlaf oder durch effektive Arbeitsweise. Die ausgebrannten Subjekte »erfahren dabei häufig eine dramatische Veränderung ihrer Zeitwahrnehmung, indem sie aus einem Zustand dynamischer oder hektischer Zeitbewegung in eine gleichsam >stillstehende ‘ Zeit fallen, die sich in eine zähe Masse verändert zu haben scheint. Jede bedeutsame Verbindung zwischen Vergangenheit, Gegenwart und Zukunft scheint dabei verlorenzugehen.«(Rosa 2013: 101 [Herv. i. O.]) Während dieser Erfahrung der Zeitwahrnehmung versuchen die Subjekte über ihre Lebensgeschichten zu grübeln mit dem Versprechen, die restliche Zeit ihres Lebens mit mehr Vorsicht, besseren Vorsätzen und weniger Ansprüchen zu verbringen. Vor allem wollen sie aber mehr Zeit für sich selbst einräumen und diese Zeit für sich selbst besser als vor dem Burnout nutzen. Diese Privatisierung der Zeit durch die Subjekte wird von einer viel globaleren Beherrschung der Zeit begleitet.

Die Prävention sorgt nämlich dafür, dass die Zeit gebraucht werden kann, dass sie für Zwecke und Ziele eingesetzt werden kann, die bestimmten Interessen mehr dienlich sind als anderen. Wenn entschieden wird, welches Verhalten, welche Berufswege, welche Politikgestaltung, welche globalen Ziele, welche Bevölkerungsgruppen bedroht sind und damit mehr Aufmerksamkeit erfordern, dann wird im gleichen Zug darüber entschieden, was, wie, wann und unter welchen Bedingungen regiert oder nicht regiert werden soll. Knappe Zeit nötigt zu Handlungen, verringert den Entscheidungsraum, beschränkt die absehbaren Optionen, reduziert die Zahl der Beteiligten, ordnet die Prioritäten um, erschöpft die Kräfte, erzeugt Nervosität.

Schließlich bleibt das präventive Handeln stets offen, denn die Zeit hat keinen Horizont, keinen definierbaren Endpunkt. Die Gefährdung muss nie, kann aber jederzeit auftreten. Ihre Vorbeugung muss daher nicht hinterfragt und ihre Relevanz nicht bestritten werden. Stattdessen kann jede nicht vorgebeugte Gefährdung eine noch größere Gefährdung hervorrufen, womit das Höchstmaß, gleichwie das Mindestmaß des Vorbeugens nicht festgelegt werden kann. Das Regieren durch Vorbeugen erstreckt sich damit einerseits auf die privatisierte Zeit des Individuums 
und andererseits auf eine allgemeine Wahrnehmung der Zeit als einer Wertmarke, gegen die die Sicherheit, Gesundheit und das Wohlbefinden der Gesellschaft ausgetauscht werden kann. Die Prävention bedient sich nicht der Zeit, sie problematisiert und beherrscht die Zeit und macht sie zum Instrument des Regierens und Regiert-Werdens, zu einer Variable gouvernementaler Technologien.

\subsection{Die Lust am Körper}

Obwohl sich die Burnout-Prävention vornehmlich auf die Stärkung und Verhütung psychischer Zustände konzentriert, erscheint der Körper des betroffenen Subjekts auf doppelte Weise bedeutsam. Zum einen werden die Anzeichen einer psychischen Schwächung von den Betroffenen wie von den Ärtzen und Therapeuten an dem Körper beobachtbar, was ihn andererseits zum Betrachtungs-, Diagnose- und Präventionsgegenstand transformiert. Damit rückt die präventive Sorge um den Körper, sein Empfinden und seine Verletzbarkeit in den Vordergrund und übt in ihm und auf ihn ihre Macht aus.

Wie Alfred Hirsch in Rückblick auf Heinrich Popitz bemerkt hat, waren sich die Autokraten und Despoten aller Länder »immer schon im Klaren darüber, dass die >Todesgefährlichkeit von Herrschaft ihre höchste Bestandsgarantie ist.« (Popitz 1992: 54 zit.n. Hirsch 2015: 193 [Herv. i. O.]) Doch nicht nur despotische und autokratische Staaten haben mit dem Körper und seiner Verletzbarkeit gespielt, sondern auch die modernen und liberalen Staaten (vgl. ebd.). Unter den liberal-demokratischen Verhältnissen wird jedoch die Verletzbarkeit des Körpers nicht nur durch direktes Schmerzempfinden, sondern auch auf indirekte Art und Weise zu Regierungszielen gebraucht. Ein Beispiel für solchen Umgang mit dem Körper bietet die Erfahrung der Einsperrung in das Gefängnis: "Sie nimmt dem Individuum die Möglichkeit, seinen Körper frei zu bewegen, frei seine Umgebung und seine Mitmenschen zu wählen. Diese Art der körperlichen Verletzung lässt sich forcieren, durch den Entzug und die Vorenthaltung von Nahrung oder den Mitteln, die notwendig sind, um diese zu erwerben.« (Hirsch 2015: 195) Ein weiteres Beispiel stellt die mehr oder weniger direkt hervorgebrachte soziale Entgrenzung der Individuen, und damit auch ihrer Körper, dar: "Was zunächst kaum den Anschein körperlicher Verletzung erweckt, kann sehr schnell dazu führen, dass Menschen hierdurch der Zugang zu lebenswichtigen Ressourcen versperrt wird.«(a.a.O.: 196) Schmerzfrei und gewaltsam zugleich wird die Macht auf und in dem Körper der Individuen auch durch das sanfte Regieren durch Vorbeugen vollzogen.

Wichtig für die gezielte Fokussierung auf den Körper war die Sensibilisierung des Bewusstseins für seine Verletzlichkeit, seine Vulnerabilität, welches »mit der Ambivalenz von Unsicherheit und Risiko, von Schmerz und Leiden auf der einen und von Leidensprävention und -eliminierung auf der anderen Seite« verbunden ist (Burghardt et al. 2017: 9). Aus einer gesellschaftskritischen Perspektive stellt 
sich in diesem Zusammenhang die Frage, wie die Bedingungen dessen hergestellt werden, wie die Körper der Subjekte zu unterschiedlichen Zeiten, unter diversen Umständen und auf besondere Art und Weise als vulnerabel dargestellt werden und sowohl den Subjekten als auch der Öffentlichkeit schutzwürdig erscheinen, und damit den Zugang zu ihrer Behandlung, Entmachtung und Prävention eröffnen. Die Vulnerabilität des Subjekts »als potentielle oder aktuelle Disposition ist immer von vulneranten >Momenten gig.« (a.a.O.: 12 [Herv. i. O.]) $)^{1}$

Prävention operiert durch die Vorstellung, dass Vulnerabilität durch Stärkung der Resilienz, Aufbau von Schutzkompetenzen, Förderung der Ermächtigung reduziert, auf Mindestniveau herabgesenkt oder gar abgeschafft werden kann. Die neuzeitliche Vorstellung der Schwächekorrektur akzeptiert keine »Kultur des Scheiterns « (Keupp 2010b: 16 [Herv. i. O.]), sondern setzt die körperlichen und psychischen Befindlichkeiten der Subjekte aufs Spiel, um die möglichen Regierungsräume ständig neu produzieren zu können. Jede festgehaltene Verletzlichkeit fordert Aufmerksamkeit, Behandlung und Prävention und bringt damit nicht selten »neue Probleme, Gefahren und Risiken« hervor (Burghardt et al. 2017: 10), die wiederum auf die Schwäche des Körpers pointieren. Die konstruierte Vulnerabilität des Körpers benennt und erzeugt damit seine Schwachstellen und behandlungsbedürftigen Partien, womit die Körper der Subjekte »einer kontingenten Ordnungslogik von diskursiven und nicht-diskursiven Elementen, von Interessen und Machtkonstellationen « ausgeliefert werden (a.a.O.: 14). Der Umgang mit Schmerz, Leid und Unglück wird an und in dem Körper selbst vollbracht, als Ausdruck einer Sorge um Vorbeugung vor noch größerer Verletzlichkeit, die ohne frühzeitige Intervention auftreten könnte. Das Machtregime der Prävention toleriert wohl eine Verletzlichkeit, doch nur eine behandelbare, eine, die den Körper und die Psyche bereitstellt, entkleidet, durchblicken und antasten lässt. So wie die Macht den Sexualitätsdispositiv bereitgestellt hat, um die Notwendigkeit der Einschränkung des Sexes zu rechtfertigen und Kontrolle über ihn zu erhalten (vgl. Foucault [1977] 1978b: 114), so entwirft sie den Präventionsdispositiv, um den kontinuierlichen Zugang zu den Körpern und ihrer Differenzierung, Beobachtung und Stigmatisierung sicherzustellen und uns glauben zu lassen, dass die körperlichen und psychischen Befindlichkeiten präventiv und kurativ lösbar sind und also so früh und so lange wie möglich - von der Pränatal- bis zur Sterbephase - überwacht werden müssen. dass es auch vulnerante, d.h. verletzende Faktoren gibt, die die Vulnerabilität hervorbringen (können).«(Burghardt et al. 2017: 12) 


\subsection{Regieren durch das Nicht-Existente}

Das immanente Regieren der Gouvernementalität, wie es von Michel Foucault und von den Denkern der Gouvernementalitäts-Studien betrachtet wird, bedient sich quantifizierbarer und messbarer Wissenselemente über Individuen oder Gruppen, die sie entweder vorfindet oder selber problematisiert. So kann das Ausbrechen der Pest dazu führen, dass hygienische Maßnahmen eingeleitet werden, die genau untersuchen, welche Regionen, Bevölkerungsgruppen oder Biosysteme besonders bedroht sind und durch welche Merkmale diese Elemente identifiziert werden können. Anhand dieses Wissens lässt sich dann ein konkretes Vorgehen entwerfen und vollziehen. Auch das Regieren des Burnouts braucht das Wissen darum, was die Subjekte empfinden, mit welchen Sorgen sie konfrontiert sind, welche Körperanzeichen ihre Störung begleiten und welche Auffälligkeiten bei ihrem Burnout auftreten. Sodann entstehen Konzepte, die sich um die Behandlung und Prävention von Burnout kümmern und genau wissen, wo sie ansetzen sollen. Allerdings, indem das Machtregime der Prävention immer mit dem Unbekannten rechnet, rechnet es gleichzeitig auch mit dem, was nicht quantifizierbar, nicht hautnah spürbar, nicht messbar und wissenschaftlich erfassbar ist, d.h. mit dem, was nicht existent ist.

Mit der Frage nach der Regierung des Nicht-Existenten wird die Schattenseite der Prävention betreten. Bisher haben sich die Analysen und die Kritik auf das zentriert, was das Regime der Prävention, zumindest analytisch, sichtbar betreibt. Es bleibt jedoch offen, was die Prävention unbewusst macht bzw. was ihr »Tun tut « (vgl. Foucault in Dreyfus \& Rabinow [1982/1983] 1994: 219), d.h. was ihre nicht-intendierten Folgen sind. Gleichzeitig stellt sich auch die Frage, wie die Prävention über das Hervorbringen von Räumen, die (noch) nicht existieren, vom Wissen, das (noch) nicht reell ist, von Möglichkeiten, die (noch) nicht vollziehbar sind, von Subjektivitäten, die (noch) keine Träger haben, regiert bzw. das Regieren wirklich und wirkmächtig macht. Dabei geht es nicht um eine Spekulation darüber, wie die Lebensweisen der Subjekte vorbestimmt und der Kontrolle immanenter Regime, wie des Präventionsdispositivs, völlig unterworfen sind. Wie Paul Veyne in Anlehnung an Michel Foucault nahelegt, ist das Dispositiv »weniger der Determinismus, der uns hervorbringt, als vielmehr das Hindernis, auf das unser Denken und unsere Freiheit reagieren oder nicht reagieren« kann (Veyne [2008] 2009: 116). Demnach soll hier die Perspektive darauf gerichtet werden, wie das Regieren über das betrieben wird, worauf die Subjekte nicht reagieren bzw. über das, was keine Kritik, keinen Widerstand, keine Auffälligkeit, kein Unbehagen hervorruft. Gelingt das Regieren auch über das Nicht-Re(-a-)gieren?

Philosophisch betrachtet betrifft das Problem der Nicht-Existenz »die >grundlegende Struktur von Denken und Sprechen« (Erhard 2017: 6 [Herv. i. O.]) und besteht im Kern darin, »dass Denken und Sprechen eine rrelationale Struktur< zu ha- 
ben scheinen.« (ebd. [Herv. i. O.]) Vor allem Edmund Husserl widmete den Großteil seiner philosophischen Bemühungen dem Problem der Nicht-Existenz, die für ihn mit der Theorie der Intentionalität zusammenhängt. Aus seiner phänomenologischen Perspektive lässt er einerseits »die Möglichkeit intentionaler Phänomene, die auf nicht-existierende Objekte gerichtet sind« (a.a.O.: 67) zu, hält aber gleichzeitig auch »an der Existenzabhängigkeit von Relationen« (ebd.) fest. Das heißt, dass intentionale Erlebnisse in sich selbst die Beziehung zur Außenwelt haben - als Wahrnehmungen existieren - unabhängig davon, ob sie sich auf ihre Existenz oder Nicht-Existenz beziehen (vgl. a.a.O.: 69). Dagegen können Relationen nur zwischen zwei reell existierenden Relationsgliedern existieren: »Ist die Beziehung, so auch die Beziehungspunkte.« (Husserl [1894/8] 1979: 420 zit.n. Erhard 2017: 68) Doch nicht nur dem Denken und Sprechen, sondern auch der Macht, wie sie von Arthur Schopenhauer, Friedrich Nietzsche und später von Michel Foucault gedacht wurde, kann ein relationaler Charakter zugeschrieben werden (vgl. Klass 2008: 152). ${ }^{2}$ Das bedeutet, dass sie nur über das ausgeübt werden kann, was existiert, d.h. zu dem eine Beziehung aufgebaut werden kann: »Wie kann nichts etwas individuieren und bestimmen? « (Erhard 2017: 70) Andererseits kann auch nur darauf reagiert und dagegen Widerstand geleistet werden, was sich auf das Subjekt bezieht, es betrifft, berührt, anspricht, eben an ihm agiert. Über das Nicht-Existente zu regieren würde dann heißen, einen Möglichkeitshorizont zu schaffen, auf den sich die Subjekte in ihren Sorgen, Vorstellungen oder Sehnsüchten beziehen könnten. Mit anderen Worten, um über die Subjekte Macht ausüben zu können, muss die Macht eine Beziehung zu ihnen schaffen, sie in Relation zu sich selbst stellen. Das Regime des Regierens durch Vorbeugen schafft Gefährdungen, die (noch) nicht existieren, Sorgen, die (noch) nicht entstanden sind, Burnout-Zustände, die (noch) nicht aufgetreten sind, damit sich die Subjekte in ihrer Selbstsorge auf sie, die nicht-existenten Möglichkeiten, beziehen und daher sich der Macht zur Verfügung stellen können.

Das Regieren durch Vorbeugen vollzieht sich also durch mindestens drei Züge: es lässt die Subjekte daran glauben, dass sie ihre Zeit haben, sie die Zeit besitzen und sie bestmöglich nutzen müssen, und zwar leistungsstark und gefährdungsbewusst; es lässt die Subjekte daran glauben, dass sie ihre Körper haben, die sie besitzen und aus ihnen die Instrumente einer erfolgreichen, ausbalancierten Lebensweise machen können; und es lässt die Subjekte schließlich daran glauben, dass sich vor ihnen Möglichkeiten erstrecken, auf die sie reagieren müssen, um dem Leben das Beste abzugewinnen und das Schlimmste zu vermeiden. Diese Art 
der Individualität, die den Subjekten seit Jahrhunderten aufgezwungen wurde, muss zurückgewiesen werden (vgl. Foucault [1982] 2005: 280), damit die Individuen, Frauen wie Männer, in ihrem Alltag wie in ihrem öffentlichen Engagement frei und gleichzeitig politisch handeln können (vgl. Wilde 2015: 106).

Mit diesen Schlussfolgerungen lässt sich klar beweisen, dass sich die BurnoutPrävention als eine immanente Regierungsweise etabliert und mannigfache Machteffekte erzeugt hat, die eine Hinterfragung und den möglichen Umsturz des neoliberalen Regierungsprojekts verhindern und die Subjekte in kontinuierlicher Selbstbeschäftigung halten sollen. Damit wurde die Eingangshypothese überprüft und mit der Diskussion der Widerstandspotentiale einige Ansatzpunkte zum Gegen-Verhalten geliefert. Außerdem hat die Analyse der Burnout-Prävention nach Michel Foucault die hohe Relevanz seines Forschungsansatzes bestätigt, der die Auswirkungen des neoliberalen Machtregimes brisant erfassen kann. Durch die umfangreiche Analyse des Präventionsdispositivs wurden konkrete Gegenstandsbereiche und diskursive Formationen erfasst und analytisch handhabbar gemacht, was eine Anwendung des Forschungsstils der Dispositivanalyse auf weitere Forschungsgegenstände und -felder motiviert. Die Untersuchung hat sich schließlich mit der Möglichkeit auseinandergesetzt, die Kritik dort zu erproben, wo sie zunächst sinnlos und problematisch erscheint, nämlich im Zustand einer völligen Erschöpfung, Macht- und Ratlosigkeit. Damit wurde versucht, die Grenzen und Möglichkeiten eines Widerstands und einer Selbstbestimmung unter unterdrückenden Umständen zu skizzieren. Hierdurch stellt sich diese kritische Arbeit in die Tradition solcher Denker wie etwa Viktor Frankl, den die Erfahrungen aus den Konzentrationslagern in der Überzeugung gestärkt haben, dass die Grenzen der Freiheit und der Macht nie vollständig bestimmt und nie eindeutig und immerwährend gelegt sind und daher in jedem Moment überprüft und verändert werden können (vgl. Frankl [1977] 2018). 
